\title{
ART-XC/SRG: Status of the X-ray optics development
}

M. Gubarev, B. Ramsey, V. Zavlin, D. Swartz, R. Elsner, S. O’Dell, K. Kilaru, C.Atkins, J. McCracken, M. Pavlinskiy, A. Tkachenko, I. Lapshov

\begin{abstract}
:
The Astronomical Roentgen Telescope (ART) instrument is a hard x-ray instrument with energy response up to $30 \mathrm{keV}$ that is to be launched on board of the Spectrum Roentgen Gamma (SRG) Mission. The instrument consists of seven identical mirror modules coupled with seven CdTe strip focal-plane detectors. The mirror modules are being developed at the Marshall Space Flight Center (MSFC.) Each module has 65 sq. cm effective area and an on-axis angular resolution of 30 arcseconds half power diameter (HPD) at $8 \mathrm{keV}$. The current status of the mirror module development and testing will be presented.
\end{abstract}

